\section{Made to order}

\section{John Mann}

The Logic of Chemical Synthesis. By E. J. Corey and Xue-Min Cheng. Wiley: 1989. Pp. 436. \$29.95, £19.15.

THE structure elucidation of complex, biologically derived molecules has attracted the attention of organic chemists since the beginning of the nineteenth century. In contrast, the construction of these molecules has been solely a twentiethcentury pursuit. Initially, the targets of these endeavours were relatively simple structures; even so, the syntheses were daunting given the paucity of starting materials and chemical reagents, and the complete absence of chromatographic and spectroscopic techniques.

Nevertheless, such complex molecules as quinine and haemin were synthesized before the Second World War. With the advent of increasingly sophisticated spectroscopic and chromatographic methods, and a new understanding of stereochemical principles and of electronic theories of mechanism, ever more challenging targets succumbed to synthesis. By 1960 the growing catalogue of achievements included syntheses of morphine, cortisone, strychnine, colchicine, penicillin $\mathrm{V}$ and chlorophyll.

All of these synthetic routes involved several steps, and some of them required heroic efforts in order to overcome pooryielding stages. Although the routes were chosen after careful consideration of the starting materials available, and with a view to employing particular (often novel) reactions, there is little evidence to suggest that a systematic analysis of the target structures was carried out. The syntheses were thus individual triumphs but not necessarily particularly efficient.

All this changed in 1967, following the publication of a seminal paper by $\mathrm{E}$. J. Corey. He exhorted chemists to analyse their problems, and introduced the concept of retrosynthetic analysis. This involves 'transformation' of the target molecule into a synthetic precursor; and these 'transforms' are just the reverse of the potential synthetic operations. Because there are usually several possible precursors, and because these in turn have numerous other progenitors, a tree of potential intermediates is easily generated. Chemists are then required to exclude those transformations for which the synthesis would (in their opinion) be difficult or impossible. In this way, the shortest route from a cheap or readily available starting material can usually be found.

It would be misleading to suggest that it was Corey alone who invented retrosynthetic analysis; but he was the first to enunciate the general principles, and he went on to provide a comprehensive set of rules and regulations. The culmination of these enquiries into the logic of synthesis was the design of a computer programme, LHASA, which represents an automated version of the analytical process.

The book reviewed here provides the first full account of 20 years of retrosynthetic analysis. It is divided into three parts. In the first 100 pages, the basic concepts and strategies are explained in full with numerous illustrative examples. This section could easily stand alone as a record of Corey's contributions to the logic of chemical synthesis. In the following 260 pages, retrosynthetic analyses (accompanied by actual synthetic schemes) are provided for 60 biologically derived molecules, and for just about all of the eicosanoids (prostanoids, leukotrienes and so on). All of the syntheses described were carried out by the Corey group. The final section includes a comprehensive literature survey of other multistep syntheses accomplished during the past 20 years.
On one level, the book undoubtedly represents a celebration of Corey's contributions to synthetic and retrosynthetic analysis - and why not! Few other synthetic chemists can claim to have devised and overseen the construction of so many interesting molecules. Some of the schemes have appeared in a similar form in other books (for example in A. Mitra's The Synthesis of Prostaglandins published by Wiley in 1977), but to have a full account of theory and practice in one place is invaluable.

This is an advanced-level volume and is not really suitable for undergraduate use. The methodology of retrosynthetic analysis is dealt with in a simpler fashion by Stuart Warren in Organic Synthesis: the Disconnection Approach (Wiley, 1982). But for anyone seriously involved in the art of organic synthesis, the book is a must - and a bargain.

John Mann is in the Department of Chemistry. University of Reading. Whiteknights, Reading RG6 $2 A D$, UK.

\section{Rocky choices}

\section{K.G. Cox}

Origins of Igneous Rocks. By Paul C. Hess. Harvard University Press: 1989. Pp. 336. \$65, £51.95.

IGNEOUS rocks originate as melts in the hot interior of the Earth. They have been erupted or intruded in all geological periods in a great variety of environments, from rift valleys to mountain chains and the ocean floor. Their origins and evolution are highly complex and difficult to study, and it is a brave author who attempts to cover them comprehensively.

Origins of Igneous Rocks is, however, a worthy addition to the recently muchenlarged range of textbooks on this topic. Comparison is invited with A. McBirney's Igneous Petrology (Freeman, Cooper 1984), A. Hall's Igneous Petrology (Longman, 1987), and M. Wilson's Igneous Petrogenesis (Unwin Hyman, 1989). All of these books have a good deal of ground in common - we might ask, do we need a new one, and what is special about this one?

Hess's book is probably the most purely geochemical and theoretically petrological of the four. It has a more extensive section on phase diagrams than the others, and gives good theoretical coverage of magmatic differentiation, principles of geochemistry and the structure of silicate melts. The main part of the book follows the traditional pattern of discussing various rock-associations in sequence, and concludes with an excellent section on lunar petrology, which is not covered in competing works. Hess deliberately does not set out to discuss the more 'geological' aspects of the subject, such as igneous rock textures (well done by McBirney) or the forms of intrusions, volcanoes and so on (a strong suit in Hall).

Hess's problem was the sheer difficulty of covering a subject in which there is an immense amount of information about a bewildering variety of rocks, and that of relating the theoretical opening chapters to the subsequent discussion of natural rocks. It can be done, but not in 300 pages. Hess solves the first problem, as others often have, by being less than comprehensive in his examples. Intra-plate volcanism is represented almost entirely by Hawaii, continental flood basalts by the Columbia River province, continental rifts by East Africa, and so on. Wilson is much more comprehensive at this stage, but she devotes a much larger fraction of her book to it.

Like most other authors who have written on igneous petrology, Hess discusses and evaluates existing petrogenetic ideas about specific rock groups. He is particularly good at identifying outstanding questions and inviting readers to form their own judgement, but as a consequence at times can seem almost indecisive. Different authors all see things slightly differently, however, and this may be where the principal value of the new work lies. It contains little that is particularly novel, but it is highly competent and well written. Igneous petrology is a complex subject to become familiar with, and to have another view on most topics is of considerable value.

K.G. Cox is in the Department of Earth Sciences, University of Oxford, Parks Road, Oxford OX1 3PR, UK. 